\title{
Pathological Fracture with Squamous Cell Carcinoma Arising from Chronic Osteomyelitis-A Case Report
}

\author{
Xi Yuan Ang, Yuet Yang Tan, Mohd Atiq Che Roselam, Mohamad Hafiz Mohmad Hassim, \\ Raeross Anak Jabul
}

Department of Trauma and Orthopedic, Hospital Bintulu, Sarawak, Malaysia

Email: angxiyuan@gmail.com

How to cite this paper: Ang, X.Y., Tan, Y.Y., Roselam, M.A.C., Hassim, M.H.M. and Jabul, R.A. (2020) Pathological Fracture with Squamous Cell Carcinoma Arising from Chronic Osteomyelitis-A Case Report. Open Journal of Orthopedics, 10 , 419-424.

https://doi.org/10.4236/ojo.2020.1012040

Received: September 28, 2020

Accepted: December 28, 2020

Published: December 31, 2020

Copyright $\odot 2020$ by author(s) and Scientific Research Publishing Inc. This work is licensed under the Creative Commons Attribution International License (CC BY 4.0).

http://creativecommons.org/licenses/by/4.0/

\begin{abstract}
Osteomyelitis had been treated and managed in an organized manner since past decade, and yet, still creates a substantial burden to entire healthcare system as a whole as its complications are exasperating. Although rare, squamous cell carcinoma could be one of the complications of chronic osteomyelitis. The aim of the work was to outline the presentation of illness and depict the carcinomatous transformation of chronic wound, based on literature review and patients operated on in the authors' department. In this case report, we would like to present a case of moderately differentiated squamous cell carcinoma in a 67 years old gentleman with 20 years history of chronic osteomyelitis. For our patient, option of amputation was chosen and no complications had been observed with the patient being fully satisfied and daily function restored after prosthesis fitting.
\end{abstract}

\section{Keywords}

Squamous Cell Carcinoma, Chronic Osteomyelitis, Amputation

\section{Introduction}

Squamous cell carcinoma is rare but severe complications of chronic osteomyelitis. Incidence of this case had been markedly reduced in recent years with improvement of the management of acute and chronic osteomyelitis. However, neglected cases of osteomyelitis still presented to us with complications including squamous cell carcinoma. The tibia and foot were the most common sites of involvement followed by femur and the ankle. Curative amputation is an effective treatment measure with proper amputation level proximal to the tumour to 
minimize the chances of metastases.

\section{Case Report}

A 67 years old gentleman with 20 years history of chronic osteomyelitis over distal third left tibia secondary to infected implant, presented to orthopaedic outpatient clinic with complaint of foul-smelling discharge from ulcers over left leg (Figure 1).

Clinically, there was a fungating ulceration over the anterior shin distal third of left leg with rolled out edges. There is a pus discharge from the ulcers with hyperkeratosis over surrounding skin. Routine investigation shows elevated infective parameter, total white cell counts 14,000, Erythrocyte Sediment Rate 55 and C-reactive protein's level rise. X-ray left tibia shows radiolucent lesion over distal one-third of shaft of tibia (Figure 2).

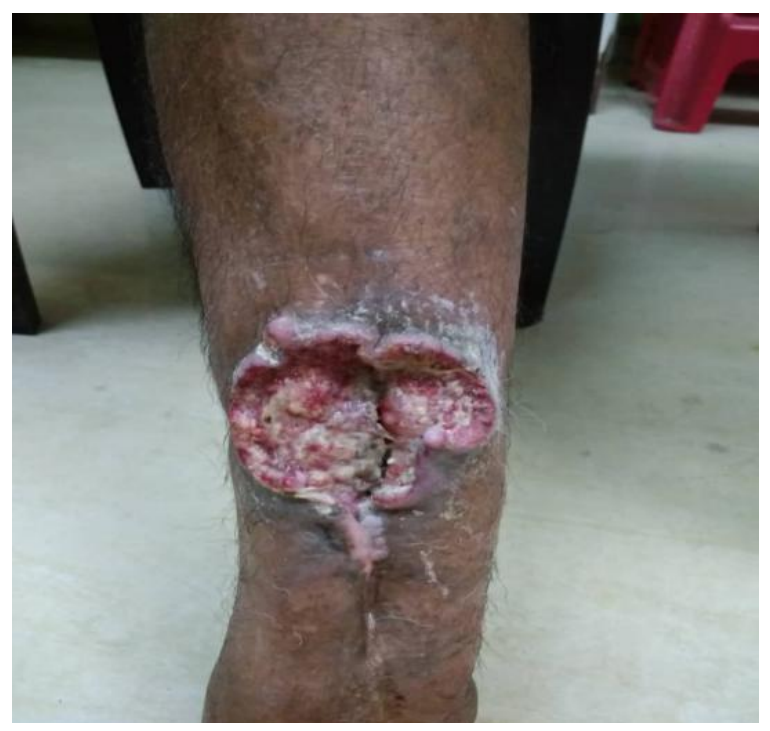

Figure 1. Shows a rodent ulcers over anterior shin left lower limb with rolled out edges and fungating base.
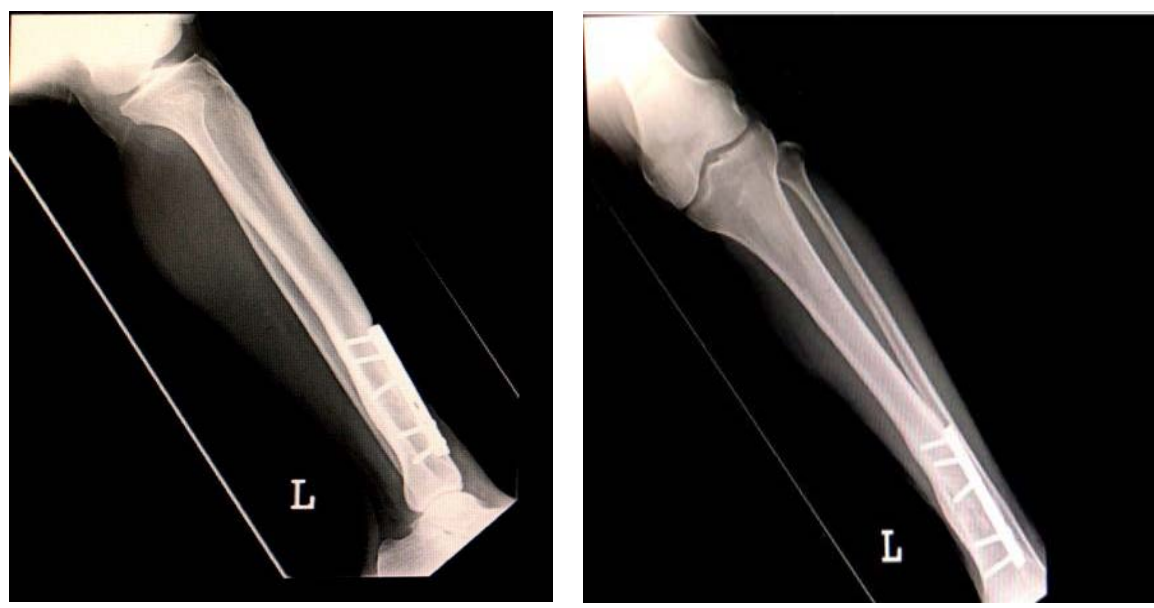

Figure 2. Radiographs reveals findings compatible with chronic osteomyelitis of tibia. 
Patient was subjected to wedge biopsy in which the biopsies were taken from the direction of 2, 5, 10 and 12 o'clock of the ulceration. Histopathological report demonstrates well-moderately differentiated squamous cell carcinoma (Figure 3). Involvement of anterior cortex of tibia beneath the wound with minimal pus discharge was noted intraoperatively.

Within 1-week post biopsy, patient re-presented to emergency department with complaint of bony pain and deformity over left leg while ambulating at home after trivial trauma. Repeated X-ray left tibia/fibula shows distal 1/3 tibia fracture with osteomyelitis changes (Figure 4).

And after careful consideration by both plastic and orthopaedic team patient was decided for below knee amputations. Burgess technique of transtibial amputation was done at level of amputation with clear margin of more than $1 \mathrm{~cm}$ proximal and depth of more than $1 \mathrm{~cm}$ from the Marjorlin ulcer's lesions.

Sample was taken from distal end of stump and was sent for histopathology examination, no malignant cells seen. The wound was well healed upon review at clinic (Figure 5).

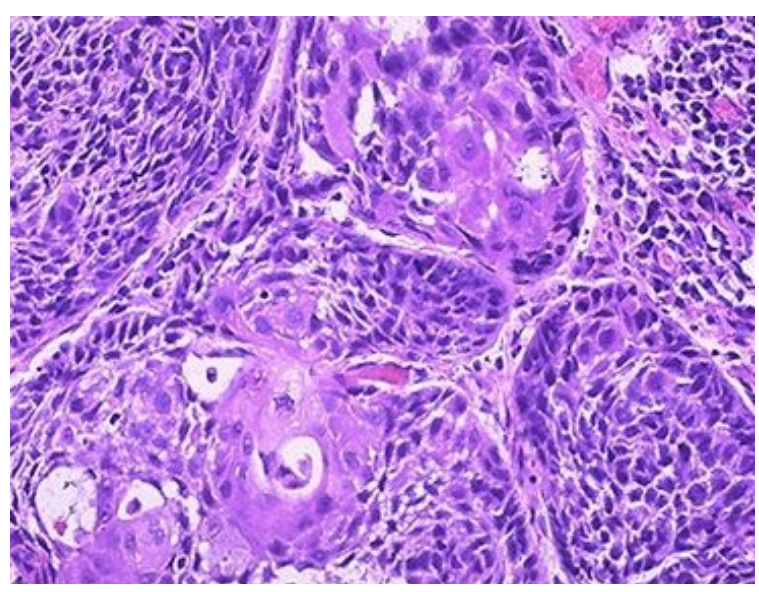

Figure 3. Well-moderately differentiated squamous cell carcinoma.

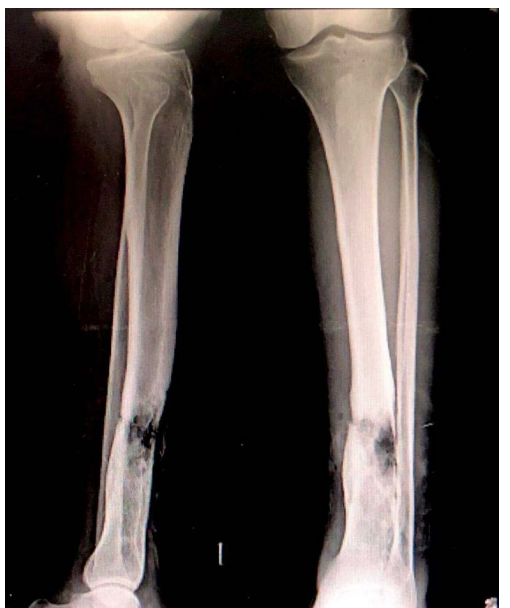

Figure 4. AP and Lateral view of distal 1/3 tibia shows pathological fracture with anterior and lateral cortex radiolucent area in late presentation. 


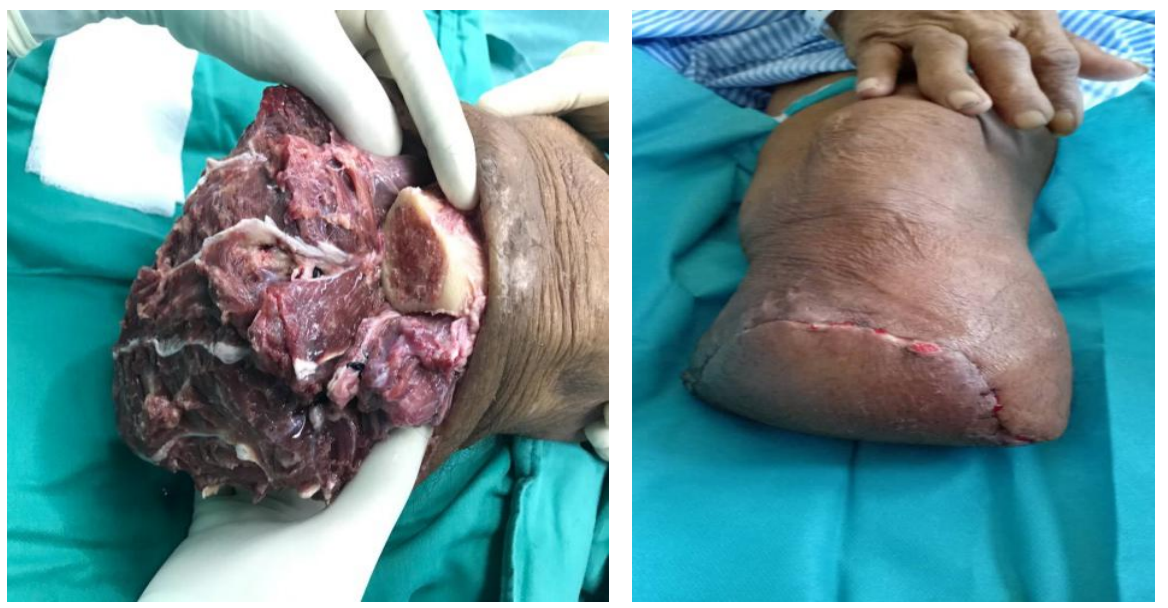

Figure 5. Healthy muscle and bone seen over the proximal stump intra operatively and wounds seen during post op examination was clean and no discharge from wounds site.

\section{Discussion}

Carcinomatous degeneration in chronic osteomyelitis is known since nineteenth century [1] with squamous cell carcinoma is the most common type apart from sarcoma or lymphoma, with incidence of skin lesions ranges between $0.2 \%$ and $1.7 \%$ of all patients affected by osteomyelitis [2]. It is rare and late complication, developing two to four decades after chronic bone infection. This degeneration may arise from fistula, particularly seen in chronic long bone infection. In contrast to squamous cell carcinoma arising de novo in otherwise normal tissue, patients with Marjolin's ulcers suffer a worse prognosis. The 5-year-survival of typical SCC, compared with Marjolin's ulcer, is $85 \%$ versus $52 \%$ respectively and the 10 -year-survival is similarly disparate, at $60 \%$ versus $34 \%$ respectively. [3]. The occurrence of metastasis is limited to $15 \%$ of cases, always occurring within five years of diagnosis [4].

History of squamous cell carcinoma often shows evidence of chronic irritation to surrounding soft tissues, including open fracture, burn injury and prolonged sun-burn in xeroderma pigmentosa predisposed group [5] [6]. Affected site includes both sun-exposed or non-sun exposed area, in which each account of different severity of the disease. In our context patient had history of trauma over extremity, proceed with open reduction and internal fixation surgery which was complicated with infected implant which leads to osteomyelitis. Despite plan of debridement offered, patient defaulted treatment. Prolonged infection over the bone and soft tissue was not treated judiciously and promptly resulting in carcinomatous changes at the osteomyelitis site.

Clinical presentations vary, including disabling pain, ulcers or sinuses, foul smelling discharge, as well as constitutional symptoms of loss of appetite and weight loss. Chronically sinus tracts of chronic osteomyelitis, are known to undergo malignant changes [7]. As the disease progress, affected bone underwent osteolytic erosion and pathological fracture as result of neoplasm infiltrating bone cortices and medullary canal, which is observed in our patient. Metastatic 
changes are not common, limited to $15 \%$. Lymphatic nodes infiltration or visceral metastasis may present, which will reduce the 5-year survival rates to $35 \%$ $50 \%$.

Baseline blood investigations with electrolytes and infective markers were taken to rule out possibility of ongoing infection and hypercalcemia that may indicate presence of metastatic evolution. Basic radiological imaging is essential to identify osteolytic changes and fracture. Biopsy over lesion is gold standard in diagnosis, staging of disease and deciding on management plan.

Amputation is widely regarded as best treatment option for advance stage of squamous cell carcinoma resulted from chronic osteomyelitis. Benjamin KJ Host in 2012 reported 2 cases of Squamouse Cell Carcinoma shows that amputation is better option of treatment as compared to en-bloc resection which requires multiple operations which were unfavorable in advance stage of carcinoma [8]. Murat Altay in 2014 also advocated amputation as best treatment option, of which five out of six study subjects were treated with amputation, despite one of the subject treated with wide resection had no local recurrence and no metastases at 136 months. [9]. Several other authors also opted for amputation in case of SCC over extrimities [2] [10] [11]. Determining factors to be taken into considerations in management plans include tumour diameter, tumour depth/level of invasion, location of tumour (sun-exposed or non-sun-exposed area), histological features, surgical margin and immune status. When diagnosed early, modern limb salvaging technique or reconstructive surgery and successful local excision have been achieved in cases of squamous cell carcinoma complicated from chronic osteomyelitis. Advanced stages with bone invasion and large skin defect, amputation is unavoidable. The reason behind amputation as choice of treatment are probably tendency of disease to metastasize early, incidence of poor results after local excision and difficulties in reconstructing the massive excised defect.

\section{Conclusion}

Squamous cell carcinoma as a complication of osteomyelitis is uncommon, yet due to the ability of malignant transformation, early diagnosis by correlations of physical examinations, imaging and histopathological biopsy is essential. Amputation is the most appropriate option for squamous cell carcinoma with chronic osteomyelitis based on previously published case report.

\section{Learning Points}

- Squamous cell carcinoma can occur many years after onset of osteomyelitis. With proper and meticulous management of osteomyelitis, occurrence of squamous cell carcinoma can be prevented.

- Marjolin ulcer with pathological fracture in patient with chronic osteomyelitis should raise suspicion of squamous cell carcinoma. Biopsy is gold standard for confirmation and grading of disease. 
- Management/surgery should be carefully decided based on clinical and histological findings.

\section{Conflicts of Interest}

The authors declare no conflicts of interest regarding the publication of this paper.

\section{References}

[1] Alami, M., Mahfoud, M., El Bardouni, A., Berrada, M.S. and El Yaacoubi, M. (2011) Squamous Cell Carcinoma Arising from Chronic Osteomyelitis. Acta Orthopaedica et Traumatologica Turcica, 45, 144-148. https://doi.org/10.3944/AOTT.2011.2537

[2] McGrory, J.E., Pritchard, D.J., Unni, K.K., Ilstrup, D. and Rowland, C.M. (1999) Malignant Lesions Arising in Chronic Osteomyelitis. Clinical Orthopaedics and Related Research, 362, 181-189. https://doi.org/10.1097/00003086-199905000-00027

[3] Wysocki, W., Komorowski, A.L., Wojewoda, T., Wysocka, J. and Mitu, J. (2010) Two Different Cases of Marjolin's Ulcer and Recommendations for Practice. The Open Surgical Oncology Journal, 2, 83-85.

[4] Bereston, E.S. and Ney, C. (1941) Squamous Cell Carcinoma Arising in a Chronic Osteomyelitis Sinus Tract with Metastasis. Archives of Surgery, 43,257-68.

[5] Marjolin, J.N. (1828) Dictionnaire de Medicine. Paris, Vol 1, 46.

[6] Stratigos, A., Garbe, C., Lebbe, C., Malvehy, J., del Marmol, V., Pehamberger, H., Peris, K., Becker, J.C., Zalaudek, I., Saiag, P., Middleton, M.R., Bastholt, L., Testori, A. and Grob, J.-J. (2015) Diagnosis and Treatment of Invasive Squamous Cell Carcinoma of the Skin: European Consensus-Based Interdisciplinary Guideline. European Journal of Cancer, 51, 1989-2007. https://doi.org/10.1016/j.ejca.2015.06.110

[7] Baitz, T. and Kyle, R.A. (1964) Solitary Myeloma in Chronic Osteomyelitis. Report of a case. Archives of Internal Medicine, 113, 872-876.

[8] Host, B.K.J., Winfield, S.J., Gollogly, J.G. and Ngiep, O.C. (2012) Marjolin's Ulcers: The Lasting Effect of Landmines in Cambodia. Asian Biomedicine, 6, 775-780.

[9] Altay, M., Arikan, M., Yildiz, Y. and Saglik, Y. (2004). Squamous Cell Carcinoma Arising in Chronic Osteomyelitis in Foot and Ankle. Foot \& Ankle International, 25, 805-809. https://doi.org/10.1177/107110070402501109

[10] Li, Q.H., Cui, H.M., Dong, J.L., He, Y., Zhou, D.S., Zhang, P. and Liu, P. (2015) Squamous Cell Carcinoma Resulting from Chronic Osteomyelitis: A Retrospective Study of 8 Cases. International Journal of Clinical and Experimental Pathology, 8 , 10178-10184.

[11] Centofanti, F. and Salfi, C. (1999) Squamous Cell Carcinoma (SCC) Arising from Chronic Osteomyelitis: A Review of 14 Cases. International Meeting on Multidisciplinary Approach to Treatment of Bone and Soft Tissue Sarcomas, 1999, Naples, Italy, 58-60. 